\title{
Textile Capacitive Sensor Arrays with Hidden Markove Model and Dynamic Time Warping
}

\author{
Shubhangi S. Marudkar ${ }^{1}$, Dr. Prashant R. Deshmukh ${ }^{2}$ \\ ${ }^{1}$ Dept. of Computer Science and Engineering, Sipna College of Engineering and Technology, Amravati, India \\ ${ }^{2}$ Professor, Dept. of Computer Science and Engineering, Sipna College of Engineering and Technology, Amravati, India
}

\begin{abstract}
Physical disabilities such as lower limb loss, manual dexterity, disable to co-ordinate with different organs of the body are very common sequel of brain injury, strokes, spinal cord injury and degenerative diseases such as affecting tissues or organs. Existing assistive technology solutions for such patients provide access as user input devices such technologies are intrusive and expensive, and require physical contact that can have hazardous effects such as skin friction injury for patients who have reduced theinzy sensitivity. To overcome this problem, in this paper, we proposed the design and implementation of non-contact proximity gessure recognition system using textile capacitive sensor arrays. The Textile capacitive sensors can be easily integrated into items of qugtation of clothing, bed sheets. This sensor is lightweight and flexible. To build this algorithm two techniques are used these are Hidor. Markovis Dynamic Time Warping. These Techniques are used to convert raw capacitance values to alphanumerifyestures. 45 system $\$$

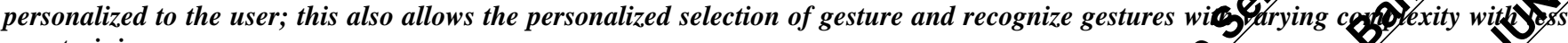
user training.

Keywords: Manual dexterity, Degenerative diseases

\section{Introduction}

Millions of individuals are hospitalized every year bentise pe plates be use

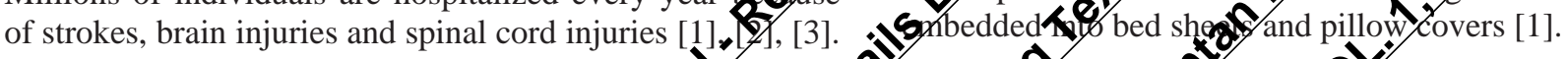
TRAUMATIC BRAIN INJURY (TBI) is apdimportant global public health problem. About 10 milligal patic are serious and results to death or hospoytization pegur annually. The patients who live with 10 are aldost the disabled persons who cannot do their Qegular wo properly [2]. Severe impairments such as paralysis, pares, weakneos and limited range of motion are common ofuels resing from these injuries and require exte(s)y rehabifirtion. These patients need assistive care devices for eny mental
control. For instance, assistive devices such switches, [4] are used for navigating whe hairs, mo calls to nurses in a hospital settingd and copohting appliances in the home. Such devices help a maximize independence and providomethods direct care [5], [6]. Gesture recogrion-base nvironm soa

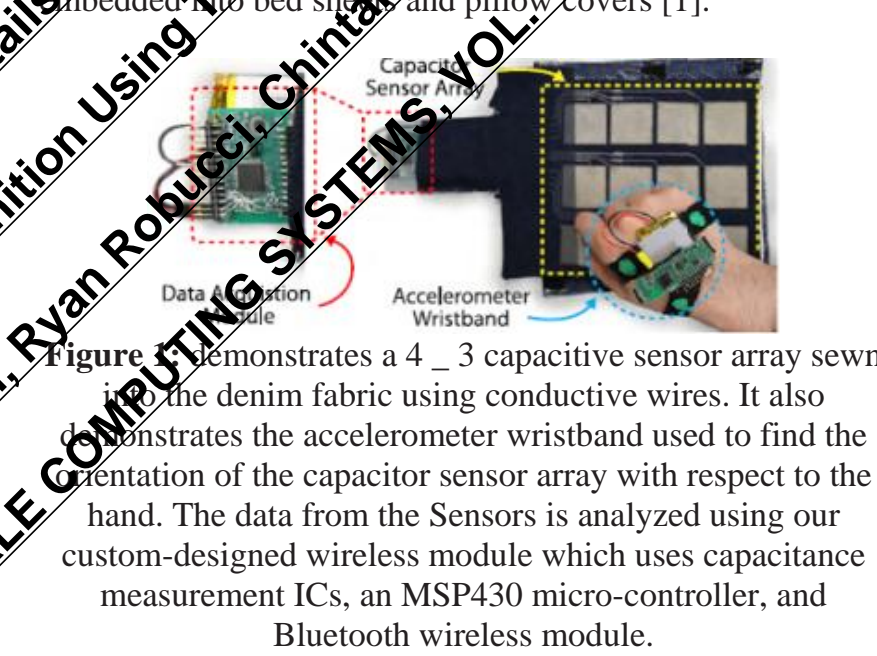
control systems are a class assistive 2 ices capas of allowing individuals with $\mathbf{Q}$ nobility 0 Pairmeks/greater control over their envirant. Sental techniats such as the use of inertial sessyrs, visio ystems, tracking can be used to capturyody gestryes [7], [6. 19], [10], [11]. Sensors for geopre recogaton are

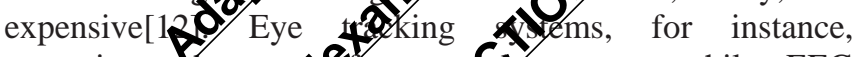
necessitate the us mound cameras while EEG electrode-based sys ems casause skin irritation and abrasion, conditions that have a deleterious effect if unnoticed due to diminjer sensation in the extremities for these individuals. In \&alty, individuals perform gestures in many different wzyn fact, the same user may perform a gesture different depending on the time of the day, medication, and fatigue. The caregivers assistive care systems should require absolute minimal setup and maintenance [1]. To Overcome all the problems we have designed, implemented and evaluated a system that uses wearable sensors built from textile capacitive sensor arrays (CSA) and a wrist worn accelerometer.[1] Our sensors work

Fig. 1 illustrates a prototype system built using the capacitive plates and conductive threads sewn into the fabric and the wrist band with an accelerometer. These sensors are proximity sensors and obviate the need for touch-based gesture recognition. An array of these sensors can be effectively used to track body positions and movements that can be consequently used to recognize gestures. We have designed and implemented a signal processing system that performs hand position tracking using the CSA, and converts a time series of hand positions into alphanumeric gestures using a Hidden Markov Model (HMM) and a model based on Dynamic Time Warping (DTW). This system learns how the gestures are performed by the user, and can adapt to different sensor configurations and placements with minimal training. The gestures are recognized irrespective of changes in orientation and placement of the sensors. 


\section{International Journal of Science and Research (IJSR) ISSN (Online): 2319-7064 \\ Index Copernicus Value (2013): 6.14 | Impact Factor (2015): 6.391}

\section{Related Work}

The spotting task of gesture recognition is much more challenging, since it is difficult to spot human motion events from number of sources. Another challenge is the system has to deal with the motion events to be spotted may only occur in a continuous data stream, while at the same time embedded into other, These movements are difficult to model due to their complexity and unpredictability. The study of gesture recognition has been continued over years and many approaches have been proposed till now. These approaches are categorized in two ways, first is gesture recognition, requiring external infrastructure and the second is gesture recognition focusing on wearable instrumentation. The first category is the vision-based motion recognition in this multiple cameras can be used for gesture recognition. The second category is started working recently and over the few period of time this has gained much attention[7]. This system which we are using in this paper is based on the second category also it is based on the previous work on capacitive sensing, gesture recognition systems and signal processing algorithms.

\section{A. Capacitive sensing technology}

Capacitive sensing technology is very popular in industrial. automotive and healthcare applications[13]. It has also beq applied to positioning [15], humidity sensing[17], zed tilt sensing[19]. This technology used to replace, Optical detection methods and mechanical design for thex applications like gesture detection and materianalysis. main advantage of the capacitive sensing yver the gesture detection approaches are that iton sense kinds of materials such as skin, p\$stic, meta pro. it is contactless and wear free, it also has the aby to seno large distance with small sensor sizes, it low coseyd low power solution. Capacitors as pro olyity sensgry have applications to robotics, industrial monitoring and thcare systems[13]. We present an end-to-end gestus s ecognitigo system including a 2D array and results for 2D tracisg and gesture classification using a domable

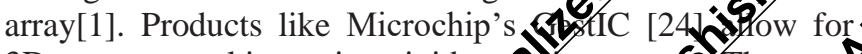
3D gesture tracking using rigid car rigidness property of our sensosflows into clothing and into the exoronment sult as bed syleets, pillow covers, and wheel ofy pads. ofitionally use of capacitive sensors (CSAllows tork colk Hotively to reduce environment hoise and apture (1) attributes like speed and dirion of moen[1]

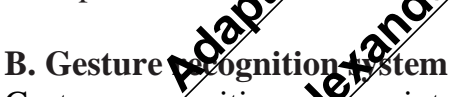

Gesture recognition sylem intery the human motion by computing device mathematisy. Gesture recognition with facial recognition, voice repognition, lip movement and eye tracking are the comprents of which developers refer as perpetual user interfa(PUI). The goal of PUI is to enhance the efficiency and the use of the underlying logical design of a stored program[1]. Most of the time the gestures are used for input commands. Recognizing gestures as input allows computers to be more accessible for the physicallyimpaired and makes interaction more natural in virtual environment. Hand and body gestures can be amplified by a controller containing accelerometers and gyroscopes to sense tilting, rotation and acceleration of movement or the computing device can be outfitted with a camera so that software in the device can recognize and interpret specific gestures. A wave of the hand, for instance, might terminate the program. The increase in adoption of home automation technologies has spawned the new platforms for control based on gestures. for instance, allow environmental control without physical contact with a controlling device. Home automation techniques have been adapted to enable users with limited mobility to have greater autonomy over environmental control. These platforms include voiceactivated systems [17], head-tracking [18], EOG based eyetracking and inertial sensors [19]. In this signal processing algorithm adapts to the user and changes in the configuration of the sensors with minimal training. Additionally, unlike systems like cameras, this system is effortlos and can be built into clothing and embedded into the syronment such as pillows and bed sheets.

\section{Signal Processing for Gestured ecognitigs}

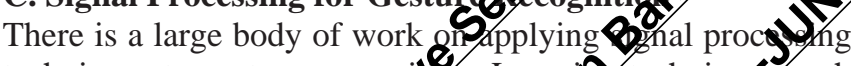
techniques to gesture recogainn. Lear technigus such as Hidden Markov Mod [20], decon tres 4 1], and Bayesian inference [2\% have bsoplaplied converting data frgm sensors Cyych as acclerometeng to movement activio and geseres. Leary approas such as HMM has also bee pplied to 9 plicationsuch as handwriting s.ognitiox 8. We . We two cystification approaches, Hidden rov Mov and Dornic Time Warping to our

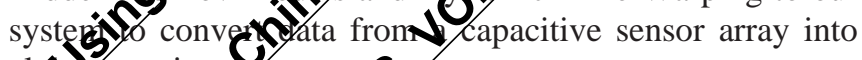

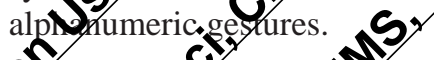

\section{Texty Capastor Sensor Array For Gesture R\&ognitiog}

gesturecognition sensor consists of textile based electrode which built into clothing or into the environment. Out goted users often lack of sensation in hands for them it (S) Critical for regulating the contact, the repeated contact be damaging to the skin to various degrees. Depending n the surface underlying. We also can create a touch-based swipe interface, in this the characters can be drawn directly on the fabric surface. The problem with this interface is that this can be develop skin friction injuries.

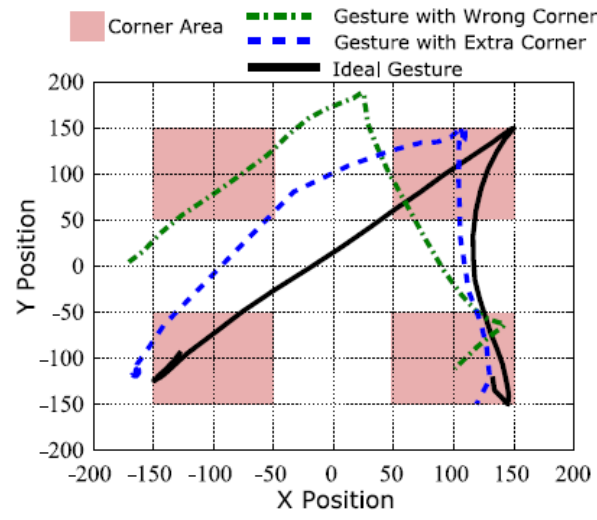

Figure 2: The gestures evaluated in this paper are based on

EdgeWrite [26] gestures, for which users are taught to envision motions though corner areas in specific orders to encourage distinct gestures. Even so, robust classification is 


\section{International Journal of Science and Research (IJSR) \\ ISSN (Online): 2319-7064 \\ Index Copernicus Value (2013): 6.14 | Impact Factor (2015): 6.391}

required. This figure depicts two situations, from three gestures within our dataset, where a state machine approach does not suffice for classification.

As fig 2. Shows the user do not always perform the exact gestures as required. Therefore we have instructed the individuals using EdgeWrite gestures set to perform distinct gestures, in order to create a robust interface so that users does not require to direct full visual attention to the interface, but still we have need the more accurate and sophisticated gesture recognition algorithm than EdgeWrite. The figure shows two situations. In this EdgeWrite state machine approach will fail due to omission of states or adding extra states. Then we presents the touch less interface, where gestures can freely performed in the proximity of the sensor. Because of this we can perform gestures with frictionless and touch less. The signals detected at the proximity have lower power and higher variance than the touch based interfaces. In the touch interface the signals are easily thresholded to create binary detection in each point of the array. Where as in touch less interfaces binary thresholding is not reliable[1]. Capacitive sensors work on the principle of change in Capacitance the electric fields between the plates of capacitor makes them highly versatile. Accelerometers and gyroscopes measure movement of the body to which they are attached. capaciti $\mathbf{\theta}$ sensors can sense movement of remote bodies.

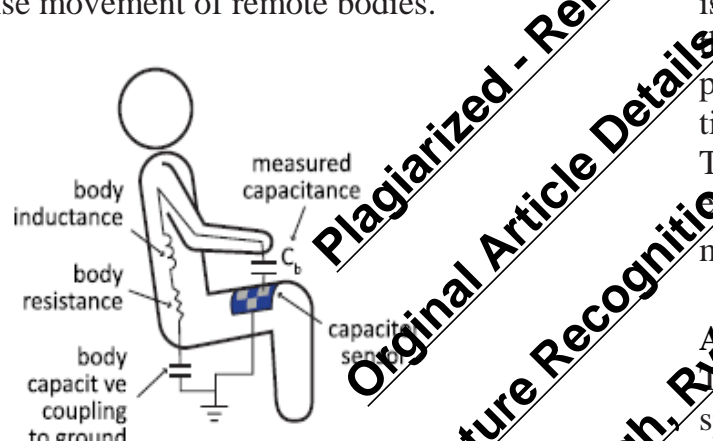
to ground to localize the hand with respect to the plates. The range of our capacitive plates is sufficient to prevent accidental touch and skin abrasion and can be adapted as the range is variable with plate size and shape. In Fig 3(b) The longitudinal crosssection of our designed sensor is illustrated The top layer is a network of capacitive sensor plates connected via conductive threads. An AC shield plane minimizes parasitic capacitance and noise coupling. The ground plane capacitively couples the human body to the ground of the sensor and provides a common reference for the capacitance measurements.

\section{Classification Algorithms for Gesture}

There are many algorithms for gesture reconition. For instance, Optical Character Recognition (OF) algorithms This is adapted for recognizing character 1 me tracked hand positions. OCR, however, views thet of pejtions as an unordered set and does not 5 variations of the tracked positionednd director of motr. Using both the temporal vagian in t.8\% data arts the direction of motion is crition our arofication the same gesture can be perfored very trently or time. In order to take advantage the ten orderif of the data,

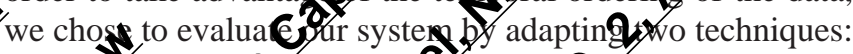

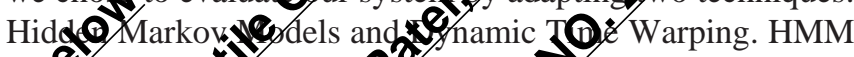

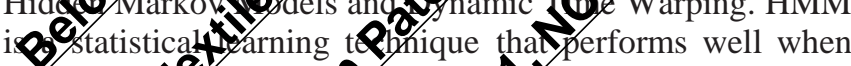
byere is variations in the Yray the gestures are performbut requ substan training and DTW is a times base 1 hnique requires minimal training. These two algoythms hel 28 evaluate trade-offs in training, Oficiency ond acculy of gesture recognition across

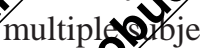

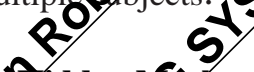

\section{AfHidden A. Skove Model}

Whe Hidd N Narkove Models are used in almost all current speechognition systems, in most of the applications such as moutational molecular biology, in data compression an $n$ other areas of Artificial Intelligence. A Hidden Markov Model is defined as a stochastic finite state machine. The algorithm is illustrated in Algorithm 1 Formally, it is defined by 5 -tuple $\Omega=(\mathrm{S}, \Sigma, \Pi, \delta, \lambda)$ where $\mathrm{S}$ represents a set of hidden states that are not directly observable. Transitions between the states is denoted by a transition probability matrix, $\delta$. $\Pi$ is the set of initial probabilities corresponding to the states in S. Every state has a set of possible emissions $\Sigma$ and continuous probabilities $\lambda$ for these emissions. The emissions can be observed by giving some information about the most similar underlying hidden state sequence which led to a particular sequence of observations. The HMM model assumes that the underlying physical process is Markovian, that means any prediction of future behavior can be optimally calculated by knowing the present state without any history. We map such a Hidden Markov Model to our problem of decoding a gesture based on a time series of hand position values. This definition of states constraints the set of gestures for which the Markovian assumption is valid. Our evaluation, however, demonstrates that this state definition works well across a large set of gestures.

without touch. The body of the patient is capacitively coupled to the ground of the sensor system. When the hand is moved close to the capacitor plates, the capacitance $\mathrm{Cb}$ increases. Inversely, the value of capacitance Cb can be used 
Algorithm1. HMM Feed Forword $(0, \Phi, \Sigma, \pi, \delta, \lambda, \mathbf{k})$

Input: Observations $(\mathrm{O}):\left\{\left(\mathrm{x}_{1}, \mathrm{y}_{1}\right), \ldots,\left(\mathrm{x}_{\mathrm{n}}, \mathbf{y}_{\mathrm{n}}\right)\right\}$ for the gesture, Hidden states (S): $\{1, \ldots . . \mathrm{k}\}$, Initial Probabilities:

$\left\{\pi_{1}, \ldots, \pi_{\mathrm{k}}\right\}$, Transition Probabilities: $\delta(\mathrm{i}, \mathrm{j}),(1 \leq \mathrm{i}, \mathrm{j} \leq \mathrm{k})$,

Emission probability distribution: $\lambda_{1}, \ldots \ldots \lambda_{\mathrm{k}}\left(\operatorname{Gaussian}\left(\mu_{\mathrm{k}}, \sigma\right)\right)$

for every state $\mathrm{k} \in[1, \ldots$ len $(\mathrm{S})]$

Output: p (Model Probability given the Observations)

for $i:=1$ to len $(O)$ do

for $k$ : $=1$ to len(S) do

Emission $=\left(1 /\left(\text { distance }\left(\left(\mathrm{x}_{\mathrm{i}}, \mathrm{y}_{\mathrm{i}}\right), \mu_{\mathrm{k}}\right) / \sigma\right)^{2}\right)+3$

for $\mathrm{j}:=1$ to len(S) do

$p(\mathrm{i}, \mathrm{k})=\mathrm{p}(\mathrm{i}, \mathrm{k})+\mathrm{p}(\mathrm{i}-1, \mathrm{j}) \times$ Emission

end for

end for

end for

return $\Sigma_{\mathrm{k}} \mathrm{p}(|\mathrm{O}|-1, \mathrm{k})$

Asymptotic Running time complexity: $\mathrm{O}\left(|\mathrm{O}| \mathrm{x} \mathrm{k}^{2}\right)$

The transition probability matrix, $\delta$ is determined during a training phase when the subject performs a set of gestures. While calculating the probability of transitions between states, we make the following key adaptation to fit our problem domain. We have found that if the sampling frequency of our sensors is large compared to the transition rate, the transition probability matrix begins to resemble an identity matrix, and the importance of transitions betwe states is diminished. The emission probabilities $\lambda$ is calculated adrordingly
Gaussian function. Each state is assigned a gosition The distance (D) from the estimated positipolnd the ceter is used as the input for the Gaussian. 50 Gaussizidth $(\sigma \omega)$ is approximately $1 / 3$ of the eeter-to-c plate distance. This width is determined and used thenghout tho experiments. For computational efficieng $\%$ we use following approximation $((\mathrm{D} / \sigma \omega)+3 \mathbf{0} \%$ the carssian. Applying the above modifications to the HMM generate a model per gesture. the gesture to be classife is pass through each of these models to create a voy of posyr probabilities relating to each Underlyide model, the model with the highest probabilith selects as the classification for the gesture.

B. Dynamic Time Warpinges/

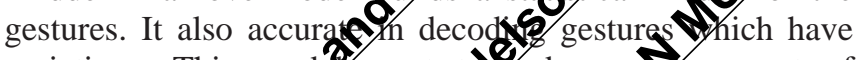
variations, This mod must Hed on

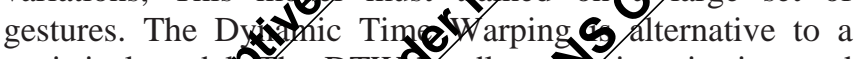
statistical modathe DT

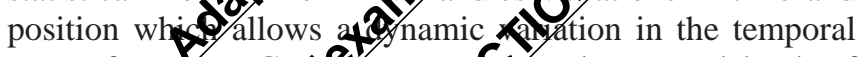
pace of gesture. Gexyes are gared to a codebook of reference gestures by find the best-case dynamic temporal distortion to cosare two gestures. Dynamic temporal distortion confrys an approach using a uniform time scaling that as the entire gesture is performed with a single speefor. This avoids requiring a reference gesture for every Wossible timing variation. Variations in the positional path are handled by providing a representative set of gesture variations in the codebook. This set is sufficient enough to classify gestures using unsupervised clustering algorithms such as a single nearest neighbor or a k-nearest neighbor. An optimal choice of dynamic time warping and minimum error is based on a euclidean distance metric.

\section{Algorithm2.DynamicTime Warping $(O, M)$}

Input: $O=\left[\left(\mathrm{x}_{1}, \mathrm{y}_{1}\right), \ldots,\left(\mathrm{x}_{\mathrm{n}}, \mathrm{y}_{\mathrm{n}}\right)\right]$ (positions for the gesture) $\mathrm{M}=\left[\left(\mathrm{x}_{1}{ }_{1}, \mathrm{y}_{1}{ }_{1}\right), \ldots,\left(\mathrm{x}_{\mathrm{n}}, \mathrm{y}_{\mathrm{n}}\right)\right]$ (model positions for the gesture)

Output: d (Warped Distance),

$\mathrm{d}(0,0)=\operatorname{distance}(\mathrm{M}(1), O(1))$

for $i:=1$ to len $(O)$ do

$\mathrm{d}(\mathrm{i}, 1)=\mathrm{d}(\mathrm{i}-1,1)+\operatorname{distance}(M(1), O(\mathrm{i}))$

end for

for $\mathrm{j}:=1$ to len $(M)$ do

$\mathrm{d}(1, \mathrm{j})=\mathrm{d}(1, \mathrm{j}-1)+\operatorname{distance}(M(\mathrm{j}), O(1))$

end for

for $\mathrm{j}:=1$ to len $(M)$ do

for $i:=1$ to len(G)do

$d(1, j)=\min _{i, j} d[d(i-1, j-1), d(i-1, j), d(i, j-1)]+\operatorname{distance}(M(j)$,

$\mathrm{O}(\mathrm{i})$ )

end for

end for

return $\mathrm{d}(\mathrm{n}, \mathrm{m})$

Complexity: $O(\mathrm{n} \times \mathrm{m})$

\section{System Evolution}

The goal of this systent to protighly a tryte gesture

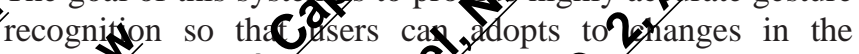
positioy and ontation of sensa) The sensor array

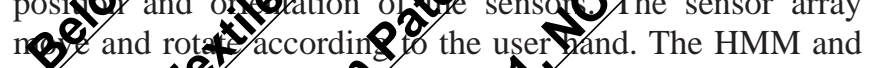

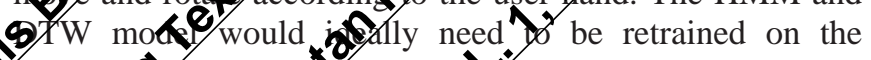

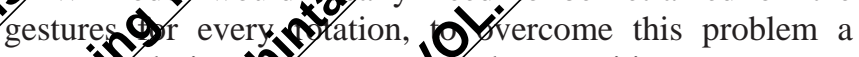
systesy designer. hat augnedyts the capacitive sensor array wit wrist wry acceles beter band, this band could be O. Haced byonlart wats

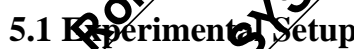

5.1 \&periments Setup

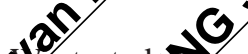

tested system on five subjects, who has C6 spinal cord injiny. For each session subject were asked to perform the 8 gestures, during the experiment ten of each type of gor 3 at different rotation of degrees of the sensor array, Ince a total 150 gestures performed per subject.

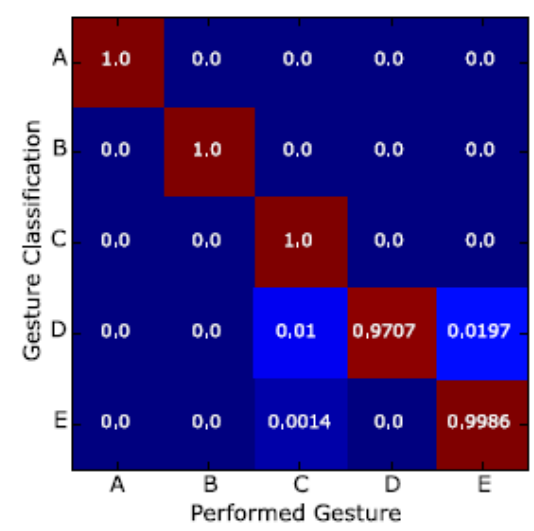

(a) DTW 


\section{International Journal of Science and Research (IJSR) ISSN (Online): 2319-7064}

Index Copernicus Value (2013): 6.14 | Impact Factor (2015): 6.391

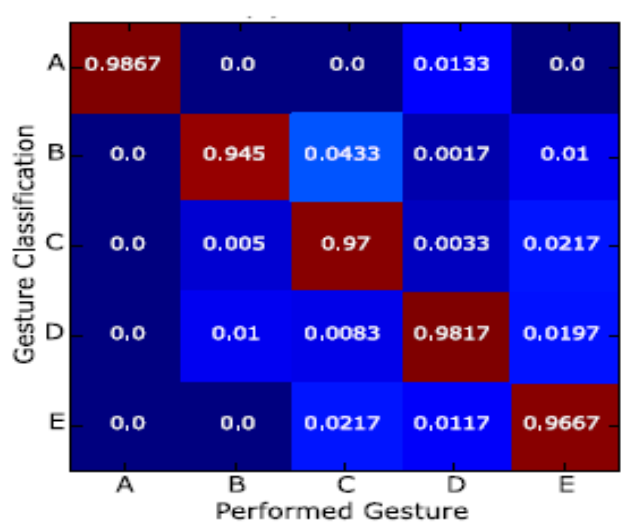

(b) HMM

Figure 4: (a) Confusion matrix illustrating the accuracy of recognizing the five gestures performed by all the subjects

when our system used Dynamic Time Warping. (b)

Confusion matrix illustrating the accuracy of recognizing the gestures performed by the subjects when our system used Hidden Markov Model. For both figures, the confusion matrix is calculated using $150 \_5=750$ gestures. Our system has an average accuracy of 99 and 97 percent for the DTW and HMM models respectively.

The figure 4 illustrate the classification of accuracy gesture recognition algorithm. The figure generated data from all gestures performed by five subjects \& 2 sensor array rotated different degrees with respect 18 hand. The average accuracy is 99 and 97 percent of 1 T $\mathrm{W}$ and HMM respectively. If there is a large variation the way gestures performed then HMM model wgroy performest while the DTW would perform poorly.

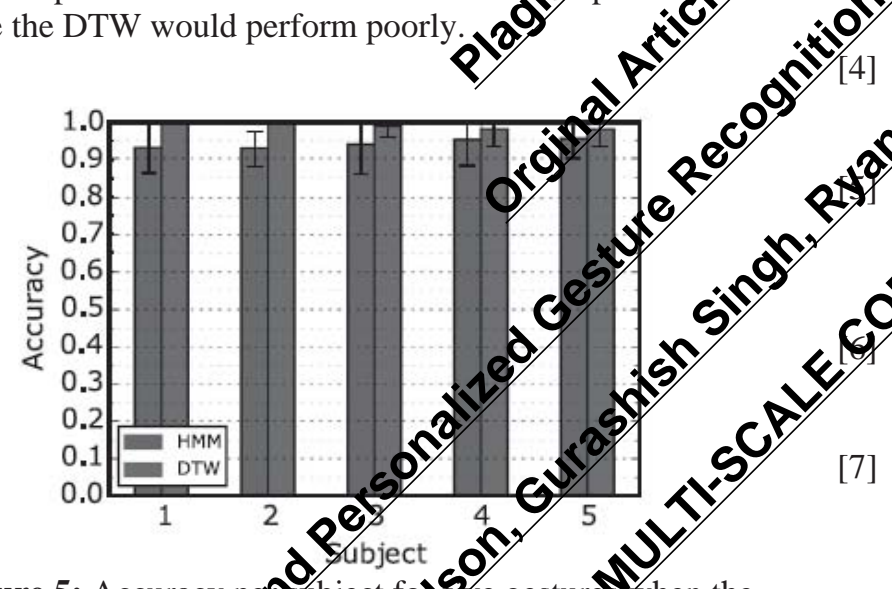

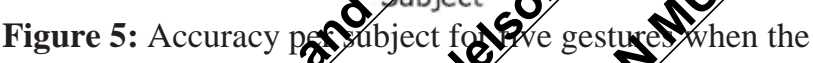
classification algoridn uses a $\$$ moder an HMM The figure based gesture recogner per sute The height bar shows the accuracy across live gest 8 s while the error bar shows the standard deviation in accuracy across gestures per subject. The focus of experiment on the subjects who has C6 spinal cord jury. The subject perform some set of gestures and it sthe the accuracy of DTW algorithm on the gestures is 99 percent , Hence we can say that this system works very well even though the subject use limited mobility.

\section{Conclusion}

In this paper we proposed a system which uses the textile capacitive sensor arrays to recognize gestures for the users who are physically disable and unable to do their routine, this system can provide gesture recognition with limited mobility. The textile sensors are flexible and can built into clothing or daily use items such as pillow covers, bed sheets, wheelchair pads etc. An adaptive and personalized signal processing system can convert capacitance data from sensor array to alphanumeric gestures. The gestures can use then to control the appliances in the home. By using the wrist-worn accelerometer and the sensor array, the gesture recognition system correct the rotation of the sensor array with the hand with minimal intervention. This system has saluated by using the HMM and DTW classification. Tho TW is more computationally efficient option.

\section{References}

[1] Alexander Nelson, Stude Member Singh, Member, IEEF $\mathbf{y}$ an Robrd, MembYIEEE,

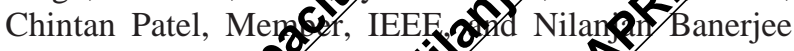
"Adaptive and Parnalized sture Reconition Using Texdile Capaci@ue Sensor Nrays" [2] A. Lang \&, W. Ruthomatic brain injury: 12 brief $0 \%$ view," J. Head Trauma Rehabil., vol. . no. 5, 19.375-378006.

[3] Syational scilal cord injuy statistical center, facts and Igures at glances SHirmingham, AL: University of Alabay a Birmi<y 2015.

4] G. O) chmeiss 5 and W. Seamone, "An assistive Qtýpment काyroller for quadriplegics," Johns Hopkins Med. J., (C) 143 , no. 3, pp. 84-88, 1979.

S. Straum, J. D. Harviel, J. H. Jaffin, and M. H. Jordr. "Lightning strike to the head: Case report," J. Ruxma Acute Care Surgery, vol. 36, no. 1, pp. 11315,1994

M. S.Workinger and R.Netsell, "Restoration of intelligible speech 13 years post-head injury," Brain Injury, vol. 6, no. 2, pp. 183-187, 1992.

[7] H. Junker, O. Amft, P. Lukowicz, and G. Tr€oster, "Gesture spotting with body-worn inertial sensors to detect user activities," Pattern Recog., vol. 41, no. 6, pp. 2010-2024, 2008.

[8] A. Y. Benbasat and J. A. Paradiso, "An inertial measurement framework for gesture recognition and applications," in Proc. Int. Gesture Workshop Gesture Sign Language Human-Comput. Interaction,2002, pp. 9-20.

[9] S.-J. Cho, J. K. Oh, W.-C. Bang, W. Chang., E. Choi, Y. Jing, J. Cho, and D. Y. Kim, "Magic wand: A handdrawn gesture input device in 3-d space with inertial sensors," in Proc. IEEE 9th Int. Workshop Frontiers Handwriting Recog., 2004, pp. 106-111.

[10]Y. Wu and T. S. Huang, "Vision-based gesture recognition: A review," in Proc. Int. GestureWorkshop Gesture-Based Commun. Human-Comput. Interaction, 1999, pp. 103-115. [11] Y. Xia, Z.-M. Yao, X.-J. Yang, S.-Q. Xu, X. Zhou, and Y.-N. Sun,

[11] "A footprint tracking method for footprint tracking method for gait analysis," Biomed. Eng.: Appl., Basis

\section{Volume 5 Issue 4, April 2016}




\section{International Journal of Science and Research (IJSR) \\ ISSN (Online): 2319-7064}

Index Copernicus Value (2013): 6.14 | Impact Factor (2015): 6.391

Commun., vol. 26, no. 1, 2014, Doi: 10.4015/S1016237214500112.

[12] S. K. Fager and J. M. Burnfield, “Patients' experiences with technology during inpatient rehabilitation: Opportunities to support independence and therapeutic engagement," Disability Rehabil.: Assistive Technol., vol. 9, no. 2, pp. 121-127, 2013.

[13] J. Cheng, O. Amft, and P. Lukowicz, “Active capacitive sensing: Exploring a new wearable sensing modality for activity recognition," in Proc. 8th Int. Conf. Pervasive Comput., 2010, pp. 319-336.

[14]H. K. Ouh, J. Lee, S.Han,H. Kim, I. Yoon, and S.Hong, "A programmable mutual capacitance sensing circuit for a large-sized touch panel," in Proc. IEEE Int. Symp. Circuits Syst., 2012, pp. 1395-1398.

[15] Y. Piao, X. Xiao, X. Wang, Q. Zhou, K. Fan, and P. Liu, "Conditioning circuit for capacitive position sensor with nanoscale precision based on AC excitation principle," in Proc. IEEE Int. Conf. Electro/Inf. Technol., 2011, pp. 1-6.

[16] P.-H. Lo, C. Hong, S.-H. Tseng, J.-H. Yeh, and W. Fang, "Implementation of vertical-integrated dual mode inductivecapacitive proximity sensor," in Proc. IEEE 25th Int. Conf. Micro Electro Mech. Syst., pp. 640-643.

[17] H. Jiang, Z. Han, P. Scucces, S. Robidoux, and Y. Sun, "Voiceactivated environmental control system persons with disabilities," in Proc. Bioeng. Conf., pp. 167-168.

[18] S. B. Kang, "Hands-free interface to a vir rá reality environment using head tracking," U.S. Py 6 210, Dec. 28, 1999.

[19] A. Nelson, J. Schmandt, P. Shyamkofar, W. Wchin, D. Lachut, N. Banerjee, S. Rolling

Varadan, "Wearable multi-sensor gesture cognitiono for paralysis patients," in Proc. IEEE Serors, 2013 018. $1-4$.

[20]A. D. Wilson and A. F. Bobick, Parametrie/idden Markov models for gesture recognition," Pattern Anal. Mach. Intell., vol. 21, no Sep. 1999.

[21] G. Fang, W. Gao, and D. Zhao, language recognition based or trees," in Proc. 5th Int. Co

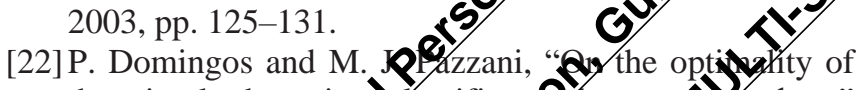
the simple bayesiandyassifier sfler zershe loss," Mach. Learn., vol.2y, no. 2-3\$p. 103-1 1997.

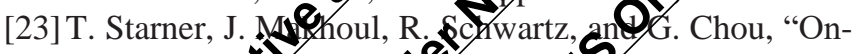
line cursivo Handwrita recogpan using speech recogniti Inethods . Speech, Dsignal Psetss., 199408. V/125-V/128.

[24] Microchip, Sin Tre-Zone Tracking and Gesture Controller Data Sheet, P. C, Nov. 2013.

[25] J. Hershberger and. Snoeyink, "An O(nlogn) implementation gre douglas-peucker algorithm for line simplific 1 ," in Proc. 10th Annu. Symp. Comput. Ge 6 try, 1994, pp. 383-384.

[26] J. O. Wobbrock, B. A. Myers, and J. A. Kembel, "Edgewrite: a stylus- based text entry method designed for high accuracy and stability of motion," in Proc. 16th Annu. ACM Symp. User Interface Softw. Technol., 2003, pp. 61-70. 